\title{
Water warming garment versus forced air warming system in prevention of intraoperative hypothermia during liver transplantation: a randomized controlled trial [ISRCTN32 I 54832] Piotr K Janicki*1, Cristina Stoica1, William C Chapman², J Kelly Wright2, Garry Walker ${ }^{1}$, Ram Pai ${ }^{1}$, Ann Walia ${ }^{1}$, Mias Pretorius ${ }^{1}$ and C Wright Pinson ${ }^{2}$
}

Address: ${ }^{1}$ Department of Anesthesiology, Vanderbilt University Medical Center, Nashville, Tennessee, 37232-4125, USA and ${ }^{2}$ Division of Hepatobiliary Surgery, Department of Surgery, Vanderbilt University Medical Center, Nashville, Tennessee, 37232-4125, USA

E-mail: Piotr K Janicki* - piotr.janicki@mcmail.vanderbilt.edu; Cristina Stoica - cristina.stoica@mcmail.vanderbilt.edu; William C Chapman - chapmanw@msnotes.wustl.edu; J Kelly Wright - Kelly.wright@mcmail.vanderbilt.edu;

Garry Walker - garry.walker@mcmail.vanderbilt.edu; Ram Pai - ram.pai@mcmail.vanderbilt.edu;

Ann Walia - ann.walia@mcmail.vanderbilt.edu; Mias Pretorius - mias.pretorius@mcmail.vanderbilt.edu; C

Wright Pinson - wright.pinson@mcmail.vanderbilt.edu

*Corresponding author

Published: 19 November 2002

BMC Anesthesiology 2002, 2:7
Received: 21 August 2002

Accepted: 19 November 2002

This article is available from: http://www.biomedcentral.com/I47/-2253/2/7

(C) 2002 Janicki et al; licensee BioMed Central Ltd. This article is published in Open Access: verbatim copying and redistribution of this article are permitted in all media for any purpose, provided this notice is preserved along with the article's original URL.

\begin{abstract}
Background: The authors compared two strategies for the maintenance of intraoperative normothermia during orthotopic liver transplantation (OLT): the routine forced-air warming system and the newly developed, whole body water garment.
\end{abstract}

Methods: In this prospective, randomized and open-labelled study, 24 adult patients were enrolled in one of two intraoperative temperature management groups during OLT. The water-garment group $(\mathrm{N}=12)$ received warming with a body temperature (esophageal) set point of $36.8^{\circ} \mathrm{C}$. The forced air-warmer group $(\mathrm{N}=12)$ received routine warming therapy using upper- and lower-body forced-air warming system. Body core temperature (primary outcome) was recorded intraoperatively and during the two hours after surgery in both groups.

Results: The mean core temperatures during incision, one hour after incision and during the skin closing were significantly higher $(p<0.05, t$ test with Bonferroni corrections for the individual tests) in the water warmer group compared to the control group $(36.7 \pm 0.1,36.7 \pm 0.2,36.8 \pm 0.1$ vs $36.1 \pm 0.4,36.1 \pm 0.4,36.07 \pm 0.4^{\circ} \mathrm{C}$, respectively). Moreover, significantly higher core temperatures were observed in the water warmer group than in the control group during the placement of cold liver allograft $\left(36.75 \pm 0.17\right.$ vs $36.09 \pm 0.38^{\circ} \mathrm{C}$, respectively) and during the allograft reperfusion period $\left(36.3 \pm 0.26\right.$ vs $35.52 \pm 0.42^{\circ} \mathrm{C}$, respectively). In addition, the core temperatures immediately after admission to the $\operatorname{SICU}\left(36.75 \pm 0.13\right.$ vs $36.22 \pm 0.3^{\circ} \mathrm{C}$, respectively) and at one $\mathrm{hr}\left(36.95 \pm 0.13\right.$ vs $36.46 \pm 0.2^{\circ} \mathrm{C}$, respectively) were significantly higher in the water warmer group, compared to the control group, whereas the core temperature did not differ significantly afte two hours in ICU in both groups.

Conclusions: The investigated water warming system results in better maintenance of intraoperative normothermia than routine air forced warming applied to upper- and lower body. 


\section{Background}

Perioperative hypothermia has been associated with morbidity in the general surgical patient population [1-6]. It is therefore appropriate to keep surgical patients normothermic (i.e., at least $36^{\circ} \mathrm{C}$ ). Despite current advances in intraoperative warming, hypothermia during the extensive abdominal surgeries, including orthotopic liver transplantation (OLT), remains often unavoidable. Treatmentresistant hypothermia during OLT is multi factorial and results from massive fluid administration, convective and evaporative losses from prolonged exposure of viscera, diminished hepatic energy production, and implantation of a cold allograft of large thermal mass [7].

A new device has been developed that circulates warm water through a special garment, which is worn by the patient during surgery and uses a microprocessor-controlled heat-pump to warm the patient to a temperature selected by the anaesthesiologist [8]. The published clinical reports about effectiveness of the water garment device in the open abdominal and cardiac surgery provided initial evidences that the intraoperative use of this system results in better maintenance of intraoperative normothermia when compared with forced air warming system. As this garment is able to cover more of the patient, and deliver heat to greater percentage of the body than forced air warming, it was hypothesized that the warming water system might also potentially prevent hypothermia during OLT more efficiently than forced air warmer.

In the current prospective and randomized trial, we compared two strategies of the perioperative maintenance of temperature using the new water warming garment with our current warming method (upper- and lower body forced-air warmer) to determine whether the new system provides more consistent maintenance of normothermia in patients undergoing OLT.

\section{Methods}

The institutional review board at Vanderbilt University approved the study protocol. In this prospective and randomized study, 24 adult patients (18-65 years old) were enrolled into the study after obtaining institutional review board accepted informed written consent. The simple randomization sequence was obtained by using a computer generated randomization list drawn up by the statistician and concealed by keeping it with the research nurse not taking direct part in the perioperative patient care. The anaesthesiologist responsible for seeing the patient in the holding area and obtaining the informed consent allocated the next available number on entry into the trial. The code was revealed to the attending anaesthesiologist and assisting research team just before transporting the patient to operating room. The control group $(\mathrm{N}=12)$ received warming therapy using a convective air warming system

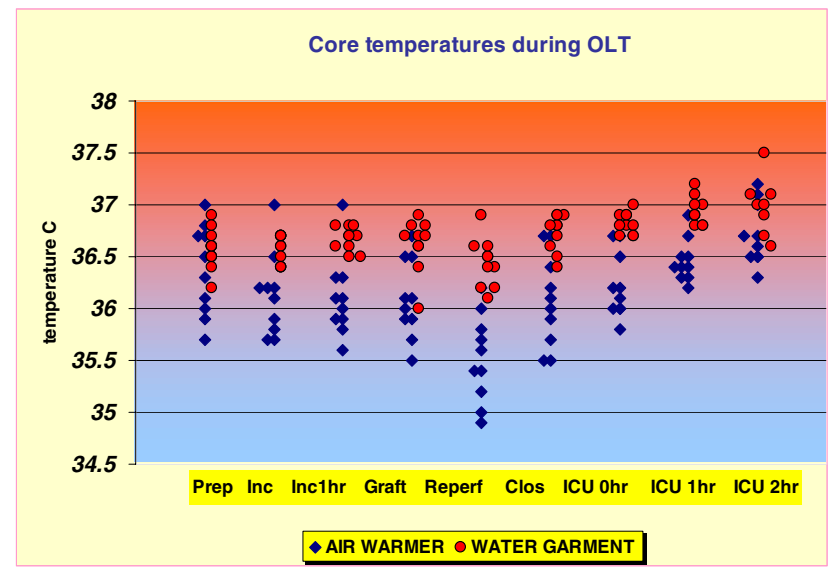

Figure I

Core temperature during the perioperative period. Data represent individual esophageal temperature points for patients in each study group. Abbreviations on horizontal axis represent different time points when the presented data were recorded: Prep $=$ OR baseline (after induction and positioning); Inc = incision; Inc $\mathrm{I} \mathrm{hr}=\mathrm{I} \mathrm{hr}$ after incision; Graft = placement of liver graft into the recipient; Reperf = reperfusion; Clos = closing; ICU $0 \mathrm{hr}=$ after arrival to Intensive Care Unit; ICU I hr= I hr postoperatively; ICU 2 hr = 2 $\mathrm{hr}$ postoperatively

consisting of two Bair-Hugger Warming Unit Model 505 and the Bair-Hugger upper and lower body warming blankets (Augustine Medical). The forced air warming unit temperature output was set on high $\left(43^{\circ} \mathrm{C}\right)$. The treatment group $(\mathrm{N}=12)$ received warming using the investigated water garment with a temperature set point of $36.8^{\circ} \mathrm{C}$. Patients were placed in the garment already pre-filled with the warm water $\left(36.8^{\circ} \mathrm{C}\right)$ before induction of anesthesia and the warming was continued introperatively until the transfer from OR table to the stretchers at the end of surgery when the garment was removed. The water warming blankets were positioned on the patient and warming started after induction of anesthesia and was continued until the end of surgery. In settings of liver transplantation surgery, we have been able to cover with the single water garment large parts of both lower and upper extremities, upper anterior, lateral portions of the chest and the entire back of the patient which accounts for about $70-80 \%$ of the total body surface. Two blankets (lower- and upper body) used in the forced air warmer system are able to cover approximately $50-60 \%$ of the total body surface.

The ambient operating room temperature was kept constant at lowest possible setting which enabled to keep the OR temperature at the range of about $20^{\circ} \mathrm{C}$ for $30 \mathrm{~min}$ before and throughout the surgery. OR temperature was continuously measured and recorded every 5 min by mer- 
Table I: Demographic of patients involved in the warming study

\begin{tabular}{|c|c|c|}
\hline & Forced Air Warmer $(\mathbf{N}=12)$ & Water Garment Warmer $(\mathbf{N}=\mid 2)$ \\
\hline Age (years) & $49.8 \pm 6$ & $5 \mathrm{I} . \mathrm{I} \pm 5(\mathrm{NS})$ \\
\hline Gender (M/F) & $7 / 5$ & $6 / 6$ (NS) \\
\hline Weight (kg) & $72.6 \pm 5.6$ & $80.1 \pm 7.9$ (NS) \\
\hline Height $(\mathrm{cm})$ & $17 \mid \pm 15$ & $168 \pm 18(N S)$ \\
\hline Preoperative temperature $\left({ }^{\circ} \mathrm{C}\right)$ & $36.44 \pm 0.4$ & $36.55 \pm 0.2$ (NS) \\
\hline \multicolumn{3}{|l|}{ Preoperative blood pressure $(\mathrm{mmHg})$} \\
\hline Systolic & $112 \pm 19$ & $108 \pm 21$ (NS) \\
\hline Diastolic & $55 \pm 9$ & $59 \pm 11(N S)$ \\
\hline Preoperative heat rate (beats/min) & $97 \pm 21$ & $89 \pm 17$ (NS) \\
\hline Ambient OR temperature $\left({ }^{\circ} \mathrm{C}\right)$ & $20.4 \pm 1.4$ & $20.4 \pm 1.5$ (NS) \\
\hline Length of operation (hrs) & $7.3 \pm 2.1$ & $6.9 \pm 1.9(\mathrm{NS})$ \\
\hline Warm ischemia time (min) & $47.2 \pm 5.9$ & $45.4 \pm 6.2$ (NS) \\
\hline Ambient SICU temperature $\left({ }^{\circ} \mathrm{C}\right)$ & $23.1 \pm 0.9$ & $22.8 \pm 0.9$ (NS) \\
\hline
\end{tabular}

Data are presented as mean $\pm S D$. NS - non significant differences between groups

cury thermometer positioned at the level of the patient and not further than $50 \mathrm{~cm}$ from the patient body. The reported mean ambient temperature represents the average of all recorded temperature measurements during the operation (for ambient OR temperature) or postoperatively (for ambient recovery temperature). No effort was made to control ambient temperature postoperatively. All intraoperative fluids were warmed intraoperatively in both groups.

For general anesthesia, standard premedication (midazolam and fentanyl) and induction techniques (i.e., thiopental, succinylcholine) were performed. Anesthesia was maintained with isoflurane, fentanyl (repeated bolus doses, as directed, as directed by cardiovascular stability, at the discretion of attending anesthesiologist) and non-depolarizing muscle relaxants (vecuronium or pancuronium) in both study groups. All patients were monitored intraoperatively using standard ASA monitors, and invasive monitors including arterial lines and pulmonary artery catheter.

An esophageal probe (Mon-a-therm ${ }^{\mathrm{Tm}}$ esophageal stethoscope 18 Fr with temperature sensor - thermistor $400 \mathrm{Se}$ ries from Mallinckrodt Corp., St. Louis, MO) was placed on the patient after induction of anesthesia. Esophageal temperatures were continuously monitored and recorded intraoperatively on the monitor console of the water warming device and a Hewlett-Packard monitoring module of the anesthesia machine. In addition, tympanic membrane temperatures were recorded preoperatively and immediately on arrival from OR to surgical intensive care unit (SICU) using a commercially available infrared probes (FirstTemp Genius, Sherwood Medical, St. Louis, MO). Upon arrival in SICU all patients were periodically assessed (every 30 min afterwards) for requirement for use of additional warming devices (warm blankets, radiant heat devices, convective air warmer). Additional warming devices were applied in the SICU in patients when they exhibited shivering or their body temperature was below $36^{\circ} \mathrm{C}$. The type of additional warming devices was left to t-he discretion of staff in SICU. The nursing staffs in SICU who were not aware of the type of thermal care received intraoperatively performed all assessments.

\section{Power analysis and statistical analysis of results}

The primary outcome from this study includes perioperative body temperatures (i.e. distal esophageal, and tympanic, when applicable) in the study and control groups. The format of this trial was expressed by two hypotheses: the null hypothesis (Ho), which states that there is no difference between control (forced air warmer) and water garment warmer group in terms of primary outcome, and the alternate hypothesis $(\mathrm{Ha})$, which states that there is a difference. A minimum sample size of 24 total subjects was calculated as needed to be enrolled and analyzed to detect a clinically relevant difference in the primary outcome of mean rectal or esophageal temperatures (temperature difference $0.5^{\circ} \mathrm{C}$ between the groups) according to the power analysis based on following parameters: type II error rate- (beta $=0.2$ ), type I error rate (alpha $=0.05, \mathrm{~d}=$ 0.5 , sigma $=0.4$ ). All non-frequency data are presented as mean SD. The parametric data were compared using twosided $\mathrm{t}$ test with the Bonferroni correction ( $\mathrm{alpha} 1=\mathrm{al}-$ $\mathrm{pha} / \mathrm{k}$ ) for multiple comparisons ( $\mathrm{k}=5$ for intraoperative comparisons and $\mathrm{k}=3$ for postoperative comparisons). The analysis on frequency data was performed using twosided Fisher. The overall statistical significance of differences (null hypothesis rejected) was inferred at $\mathrm{p}<0.05$. 


\section{Results}

There were no statistically significant differences ( $\mathrm{p}>$ 0.05 ) in the demographics (age, weight, height, sex, preoperative temperatures, blood pressure, heart rate) and no statistically significant differences in the length of the procedure and ambient OR and SICU temperatures between two groups of patients. All 24 patients underwent liver transplantation using the piggy-back technique, and no veno-venous bypass was used during the surgical procedure. No statistically significant differences between investigated groups were noted regarding the total length of surgery and anesthesia, length of warm ischemia ( $p$ > $0.05)$. The air warming blankets in the forced air warmer group were applied to the patients after induction of anesthesia and completion of the invasive line placement, whereas water warming blankets were applied to the patients in the water warming group before induction of anesthesia ( $48 \pm 16$ min time difference between the study groups).

Patients in the water warmer group maintained more consistently normothermia intraoperatively (Fig. 1). The mean core temperatures during incision, 1 hour after incision and during the skin closing were significantly higher (overall significance level $\mathrm{p}<0.05$ by $\mathrm{t}$ test with Bonferroni correction for the individual comparisons) in the water warmer group compared to control group (36.7 \pm 0.1 , $36.7 \pm 0.2,36.8 \pm 0.1$ vs $36.1 \pm 0.4,36.1 \pm 0.4,36.07 \pm$ $0.4^{\circ} \mathrm{C}$, respectively). Moreover, significantly $(\mathrm{p}<0.05 \mathrm{t}$ test with Bonferroni correction) higher core temperatures were observed in water warmer group than in control group during the placement of cold liver allograft (36.75 \pm 0.17 vs $36.1 \pm 0.4^{\circ} \mathrm{C}$, respectively) and during the allograft reperfusion period $\left(36.3 \pm 0.3\right.$ vs $35.5 \pm 0.4^{\circ} \mathrm{C}$, respectively).

The core temperatures immediately after admission to the SICU ( $36.7 \pm 0.1$ vs $36.2 \pm 0.3^{\circ} \mathrm{C}$, respectively, $\left.\mathrm{p}<0.01\right)$ and at $1 \mathrm{hr}\left(36.9 \pm 0.1\right.$ vs $36.5 \pm 0.2^{\circ} \mathrm{C}, \mathrm{p}<0.01$ respectively) were significantly higher in the water warmer group, compared to the control group, whereas the core temperature did not differ significantly at $2 \mathrm{hr}$ in ICU (36.7 \pm 0.3 vs $36.9 \pm 0.3^{\circ} \mathrm{C}, \mathrm{p}>0.05$, respectively) in both groups.

No statistically significant differences $(\mathrm{p}>0.05$, not shown) in the recorded postoperative vital signs (blood pressure, heart rate, respiratory rate and $\mathrm{SpO}_{2}$ ) were observed in both investigated groups in SICU for 2 hours postoperatively.

\section{Discussion}

Body temperature has been more consistently maintained at greater than $36^{\circ} \mathrm{C}$ during the entire OLT period (including reperfusion) and immediately postoperatively in the water warmer group compared to the patients treated with the routine convective warming methods. One possible explanation for the differences in the temperatures observed in the current study might be that the water garment warmer allows for the slightly greater body area to be covered (and warmed) compared with the double forced-air system. However, it is unlikely that the small difference in the area covered (about 20\% body surfaces, consisting of the posterior aspect of the trunk and legs) between two warming systems will offer the full explanation for the observed effects. Another reason for the observed differences in core temperatures between two devices at incision and $1 \mathrm{hr}$ after incision might be that water-garment group was prewarmed (i.e. patients were placed on the warm water garment before induction) and the airwarming group was not. It is well known that prewarming patients before induction of anesthesia reduces the coreto-peripheral temperature gradient and decreases the redistribution hypothermia, as was demonstrated before by other investigators. It is important to note that the forcedair warming of the upper-body blanket was only started after completion of placement of all invasive lines and positioning (particularly extensive during OLT), which was significantly later than the start of warming with the new water garment. It is possible, that if the two systems were compared in similar settings, these small differences in the core temperature will be no longer observed. In this respect, the current study did not compare the warming effectiveness of two different devices, but two different strategies of perioperative warming. However, in the well designed clinical comparison of these two devices in healthy hypothermic and vasoconstricted subjects, Taguchi (2002) reported that the heat transfer was significantly improved during active warming with the investigated water warming system compared to forced-air warming and the associated core temperature increase rate was also significantly faster in the water warming group [9]

The clinical value of the better control of perioperative normothermia during OLT is unclear. It has been reported recently that relatively small perioperative differences $\left(0.5^{\circ} \mathrm{C}\right)$ in core temperature were associated with significant differences in blood loss during orthopedic surgery [10]. There is now also considerable evidence that even mild perioperative core hypothermia causes numerous adverse outcomes including myocardial morbidity, coagulopathy, increased transfusion requirement, wound infection, increased recovery time and prolonged hospitalization [1-6]. More significantly, in regard to liver transplantation, it was demonstrated recently that intraoperative hypothermia during liver transplantation increases the risk for early cytomegalovirus infection in liver transplant recipients and active warming seems to reduce this risks [11]. In this respect, Nesher et al demonstrated recently that patients warmed with the investigated water 
warming garment during the off-pump coronary bypass surgery demonstrated reduced perioperative levels of interleukin 6 and 10 levels, suggesting enhanced immune response to perioperative stress compared to the routine thermal care group [12].

The comparison of the investigated water warming device with the routine (i.e. based mostly on the forced air warmer) thermal care was a subject of many, recently published investigations, including pediatric surgery with and without cardiopulmonary bypass [13-16]. In most of these investigations (including ours), the moderate $\left(0.6-1.5^{\circ} \mathrm{C}\right)$ higher core temperature was observed intraoperatively and early postoperatively in the subjects warmed with the water warming system compared to the routine warming methods. It is important to mention at this point that in most countries, the current costs of using the water warming system is considerably higher than routine temperature care, (e.g. forced air warming system). Therefore, the question remains, if the observed differences in the core temperature between investigated systems will provide clinical justification for using the more expensive system. Our study was not designed as the cost comparison study; therefore, it would be impossible to make any suggestions if the use of the particular warming system might be costeffective in any particular clinical situation.

\section{Conclusions}

The investigated water warming system, by virtue of its ability to deliver heat earlier (i.e. before and immediately after induction) to a greater percentage of the body surface in OLT patients, results in better maintenance of intraoperative normothermia than does routine forced-air warming applied to the upper- and lower body parts.

\section{Competing interests}

Study supported by unrestricted grant from MTRE Advanced Technologies Ltd, Or-Akiva, Israel. Neither author serves as paid consultant for the above company, nor has any author any direct stock holding in the above company.

\section{Authors' contributions}

PKJ conceived of the study, and participated in its design and coordination, as well as the statistical analysis of the data. CS, GW, RP, AW, MP carried out the protocol, inclusion of patients, anesthesia and data collection. WC, JKW, CWP MP carried out the protocol, inclusion of patients, surgery and data collection. All authors read and approved the final manuscript.

\section{Acknowledgements}

The authors thank the faculty, nursing and technical staff and residents in the Department of Anesthesiology, Vanderbilt University Hospital, Nashville, Tennessee, for assistance and support in performing the study.

\section{References}

I. Beilin B, Shavit Y, Razumovsky J, Wolloch Y, Zeidel A, Bessler H: Effects of mild perioperative hypothermia on cellular immune responses. Anesthesiology 1998, 89: I I33-I I40

2. Lenhardt R, Marker E, Goll V, Tschernich H, Kurz A, Sessler DI, Narzt $\mathrm{E}$, Lackner F: Mild intraoperative hypothermia prolongs postanesthetic recovery. Anesthesiology 1997, 87: I 3 18-1323

3. Schmied H, Kurz A, Sessler DI, Kozek S, Reiter A: Mild intraoperative hypothermia increases blood loss and transfusion requirements during total hip arthroplasty. Lancet 1996, 347:289-292

4. Kurz A, Sessler DI, Lenhardt RA: Perioperative normothermia to reduce the incidence of surgical-wound infection and shorten hospitalization. Study of wound infections and temperature group. N Engl J Med 1996, 334: I 209-1215

5. Sessler DI: Temperature Monitoring. In: Anesthesia (Edited by: Miller RD) Philadelphia: Churchill Livingstone 2000, I367-I389

6. Frank SM, Fleisher LA, Breslow MJ, Higgins MS, Olson KF, Kelly S, Beattie C: Perioperative maintenance of normothermia reduces the incidence of morbid cardiac events. A randomized clinical trial. JAMA 1997, 277: I I 27- I I 34

7. Russell SH, Freeman JW: Prevention of hypothermia during orthotopic liver transplantation: comparison of three different introperative warming methods. Br J Anaesth 1995, 74:4 I5-4I8

8. Janicki PK, Higgins MS, Janssen J, Johnson RF, Beattie C: Comparison of two different temperature maintenance strategies during open abdominal surgery: Upper body forced-air warming versus whole body water garment. Anesthesiology 200I, 95:868874

9. Taguchi A, Ratenaraj J, Sharma N, Nagele A, Kurz A: Evaluation of new automatic body temperature control system. Anesthesiology 2002, 96:A588

10. Winkler M, Akca O, Birkenberg B, Hetz H, Scheck T, Arkilic CF, Kabon B, Marker E, Grubl A, Czepan R, Greher M, Goll V, Gottsauner-Wolf F, Kurz A, Sessler DI: Aggressive warming reduces blood loss during hip arthroplasty. Anesth Analg 2000, 91 :978984

II. Paterson DL, Staplefeldt WH, Wagener MM, Gayowski T, Marino IR, Singh N: Intraoperative hypothermia is an independent risk factor for early cytomegalovirus infection in liver transplant recipients. Transplantation 1999, 67: I I5 I-II55

12. Nesher N, Insler S, Sheinberg N, Froilkis I, Uretzki G: Maintenance of perioperative normothermia affects immune response regulation during off-pump coronary artery bypass (OPCAB) surgery. Anesthesiology 2002, 96:A504

13. Motta P, Diego TM, Lozano S, Insler SR, Mossad E: Effectiveness of water circulating warming garment in rewarming after pediatric cardiac surgery employing hypothermic cardiopulmonary bypass. Anesthesiology 2002, 96:Al28

14. Katz J, Steinberg R, Kachko L, Metzner J, Gal MT: Novel feedback controlled circular water warming system (Allon 200I) is superior to forced warmed air device in maintaining normothermia in infants undergoing major surgery. Anesthesiology 2002, 96:A591

15. Nesher N, Wolf T, Uretzky G, Oppenheim-Eden A, Yussim E, Kushnir I, Shoshany G, Rosenberg B, Berant M: A novel thermoregulatory system maintains perioperative normothermia in children undergoing elective surgery. Paediatr Anaesth 2001, I I:555-560

16. Nesher N, Wolf T, Kushnir I, David M, Bolotin G, Sharony R, Pizov $R$, Uretzky G: Novel thermoregulation system for enhancing cardiac function and hemodynamics during coronary artery bypass graft surgery. Ann Thorac Surg 200I, 72:S1069-76

\section{Pre-publication history}

The pre-publication history for this paper can be accessed here:

http://www.biomedcentral.com/1471-2253/2/7/prepub 